\title{
EDITORIAL
}

\section{Transanal irrigation: another hope for patients with LARS}

\author{
P. Christensen ${ }^{1} \cdot$ N. S. Fearnhead ${ }^{2} \cdot$ J. Martellucci ${ }^{3}$
}

Published online: 7 October 2020

(c) Springer Nature Switzerland AG 2020

The number of long-term cancer survivors has tripled over the last 3 decades. It is estimated that there will be 4 million cancer survivors in the UK in 2030. One out of four cancer patients report chronic physical problems that significantly impair their quality of life. Gastrointestinal symptoms are most common side-effect of cancer treatment and have the greatest impact on quality of life.

The potential for poor functional outcomes comes into sharp focus with treatment for rectal cancer. The introduction of total mesorectal excision revolutionized treatment and substantially improved local control and survival. Preoperative chemoradiation (CRT) and preoperative shortcourse radiotherapy further significantly reduce recurrence. However, oncological resection of the rectum and restoring bowel continuity, with or without neoadjuvant therapy, is not without consequences for the patient. Numerous studies have reported that $50-80 \%$ of patients develop low anterior resection syndrome (LARS) characterized by increased frequency of defecation, urge, faecal incontinence, difficulty in discriminating between flatus or stool, and incomplete rectal evacuation. Living and coping with LARS has a profoundly negative impact on overall quality of life.

Recently, a large international consensus trilingual Delphi process with patients as the major stakeholder defined LARS as having at least one of eight symptoms resulting in at least one of eight consequences (see Table 1) after anterior resection [1]. Increasing research has investigated the complex pathophysiology and we now know that the aetiology of LARS is multifactorial with a combination of

P. Christensen

petchris@rm.dk

1 Danish Cancer Society Centre for Research on Survivorship and Late Adverse Effects After Cancer in the Pelvic Organs, Department of Surgery, Aarhus University Hospital, Aarhus, Denmark

2 Department of Colorectal Surgery, Cambridge University Hospitals NHS Foundation Trust, Cambridge, UK

3 General, Emergency and Mini-Invasive Surgery, Careggi University Hospital, Florence, Italy loss of reservoir function in the neorectum, biomechanical abnormalities in the neorectum and distal colon, afferent sensory abnormalities from the remnant anal mucosa, and radiotherapy-induced small bowel enteropathy and impaired anal sphincter function after multimodal therapy.

With the introduction and widespread use of the LARS score [2] as an easy-to-use short form questionnaire to screen for LARS, now validated in many languages, we are able to identify rectal cancer survivors with life-impacting LARS who need treatment. Unfortunately, despite growing awareness in recent years and focus on the quality of cancer survivorship, management of debilitating LARS is often empirical and symptom based, using existing therapies for fecal incontinence, fecal urgency and rectal evacuatory disorders.

As the search continues for potentially effective treatments, transanal irrigation may offer some hope to patients with LARS. Transanal irrigation (TAI) has been extensively studied for neurogenic bowel dysfunction and other functional bowel problems. Preliminary results suggested potential benefit in patients with LARS [3]. After initial encouraging results, transanal irrigation was included alongside rehabilitative techniques as second-line conservative treatment for LARS patients, after failure of conventional supportive care, in a management algorithm for effective management of LARS.[4].

In recent years, some studies published in this journal have further explored the potential role of TAI for the management of LARS [5, 6], and have confirmed that that TAI is effective in reducing bowel movements, episodes of urgency, and clustering and night defaecations. Introduction of TAI in patients with LARS signals significant benefit to both symptoms and quality of life. In the study of Martellucci et al., patients after a rectal resection with a postoperative major LARS score $(>30)$ were enrolled. During TAI, the median LARS Score fell from 35.1 to $12.2(p<0.0001)$ and at the end of the study, $85 \%$ of patients have chosen to continue the treatment. Most interestingly, benefits of TAI are observed irrespective of whether TAI is started early after 
Table 1 International consensus definition of LARS

\begin{tabular}{ll}
\hline Symptoms of LARS & Consequences of LARS \\
\hline Variable unpredictable bowel function & Toilet dependence \\
Altered stool consistency & Preoccupation with bowel function \\
Increased stool frequency & Dissatisfaction with bowels \\
Repeated painful stools & Strategies and compromises \\
Emptying difficulties & Impact on mental and emotional well-being \\
Urgency & Impact on social and daily activities \\
Incontinence & Impact on relationship and intimacy \\
Soiling & Impact on role, commitments and responsibilities
\end{tabular}

LARS is defined as one or more symptoms with one or more consequences following anterior resection the closure of diverting ileostomy or after many years of LARS symptoms.

In the study of Rosen et al., patients after rectal resection and stoma closure were randomly assigned to TAI + supporting therapy or supportive therapy only (ST), regardless of the LARS score or other functional evaluation values. After 12 months of follow-up, $>50 \%$ of patients continued with TAI, showing a lower number of defecation episodes per daytime ( $p$ 0.018) and per night ( $p$ 0.004) compared to the supporting therapy group. However, although the LARS score was lower in patients who used TAI, this failed to reach the level of significance ( $p$ : 0.063). Moreover, evaluation of the Wexner score and the 36-item Short Form Health Survey failed to find any statistically significant difference between TAI and ST. These results may suggest that patients with a more severe dysfunction could benefit more from the use of the TAI and for patients with a less severe dysfunction, the use of TAI may not be necessary.

So where do clinical researchers interested in this complex field go next? While increasing evidence accumulates that transanal irrigation is an effective therapy for selected LARS patients, numerous questions persist and require further research. We need to better understand which patients may benefit, develop technological advances in irrigation delivery mechanisms optimise the education for patients using, and healthcare professionals delivering TAI. Clinicians acknowledge that symptoms of LARS may diminish during the first year after restoration of bowel continuity. The question remains whether this slow recovery may be accelerated by early introduction of TAI. Further research is also needed on optimising and probably personalising volume of irrigation fluid, algorithms for troubleshooting if TAI is not working, and what to do next in case of TAI fails to improve the patient's symptoms.

Most importantly, increasing understanding of LARS and rapidly accruing clinical expertise calls for a re-thinking of the follow-up strategy with improved focus on late morbidity and possibly its prevention through early rehabilitation. These issues should be highlighted at the preoperative information and counselling of patients. Postoperative surveillance should actively incorporate assessments of function and quality of life, and not just focus on cancer recurrence. Identification of LARS allows intervention, since the majority of LARS symptoms are often improved or cured with effective treatment such as transanal irrigation.

\section{Compliance with ethical standards}

Conflict of interest The authors declare that they have no conflict of interest.

Ethical approval This is an editorial. No results are presented.

Informed consent No informed consent needed.

\section{References}

1. Keane C, Fearnhead NS, Bordeianou L et al (2020) International consensus definition of low anterior resection syndrome. Colorectal Dis 22:331-341

2. Emmertsen KJ, Laurberg S (2012) Low anterior resection syndrome score: development and validation of a symptom-based scoring system for bowel dysfunction after low anterior resection for rectal cancer. Ann Surg 255:922-928

3. Juul T, Christensen P (2017) Prospective evaluation of transanal irrigation for fecal incontinence and constipation. Tech Coloproctol 21:363-371

4. Martellucci J (2016) Low anterior resection syndrome: a treatment algorithm. Dis Colon Rectum 59:79-82

5. Martellucci J, Sturiale A, Bergamini C et al (2018) Role of transanal irrigation in the treatment of anterior resection syndrome. Tech Coloproctol 22:519-527

6. Rosen HR, Boedecker C, Fürst A, Krämer G, Hebenstreit J, Kneist W (2020) "Prophylactic" transanal irrigation (TAI) to prevent symptoms of low anterior resection syndrome (LARS) after rectal resection: results at 12-month follow-up of a controlled randomized multicenter trial. Tech Coloproctol. https:// doi.org/10.1007/s10151-020-02261-2

Publisher's Note Springer Nature remains neutral with regard to jurisdictional claims in published maps and institutional affiliations. 